\title{
Immunotherapy and hepatocellular carcinoma
}

\author{
Timothy Allen*, Nepton Sheikh K, Shoja ER and Naveed Basha C \\ Global Allied Pharmaceuticals, Center for Excellence in Research and Development, 160 Vista Oak Dr. Longwood, FL 32779, USA
}

\begin{abstract}
Hepatocellular carcinoma (HCC) is considered the most common primary cancer of the liver. HCC accounts for $90 \%$ of all liver cancers.HCC is the fifth common cause of cancer in men and seventh in women worldwide. It is the second most common cause of cancer death worldwide. It is rare in developed countries and prevalent in developing ones. Its main causes are viral infections, such as HBV and HCV. Various cell signaling pathways are demonstrated to play a role in its molecular pathophysiology. Annualy, an approximately of 500,000 deaths per year were reported. Prognosis of HCC is poor because late presentation with large tumours and also the lack of medical expertise and facilities in high HCC prevalence regions in the world. Recently Metastatic or unresectable hepatocellular carcinoma prognosis improved with immunotherapy. Immunotherapt has proven to be effective in the treatment of hepatocellular carcinoma. We review the available drugs and vaccines tested in the management of Hepatocellular carcinoma. Some has become standard of care, but many are under clinical trials with much anticipated results. In this paper we provide an overview of the class of Monoclonal Antibodies (MAB), Vaccines, mTOR inhibitors,Proteasome inhibitors,oncolytic virus therapy,cytokines, STAT3 inhibitors, check point inhibitors, Adoptive T-cell Therapy,A3 Receptor inhibitors, Myc inhibitors and kinase inhibitors in the treatment of Hepatocellular carcinoma.
\end{abstract}

\begin{abstract}
Abbreviations: HCC: Hepatocellular carcinoma; PTEN: Phosphatase and tensin homolog; FISH: fluorescence in situ hybridization; TAA tumor-associated antigen; rhGM-CSF recombinant human granulocyte macrophage-colony stimulating factor; STAT3 signal transducer and activator of transcription 3; TP53: tumor protein $\mathrm{p} 53$; TK: tyrosine kinase; VEGF: vascular endothelialgrowth factor; KDR: Kinase insert domain receptor; HER: human epidermal growth factor receptor; EML4: echinoderm microtubule-associated protein-like 4 gene; EGFR: epidermal growth factor receptor; RET: rearranged during transfection; MAPK1: mitogen-activated protein kinase 1; MET: hepatocyte growth factor receptor; PI3K: phosphatidylinositol-4,5-bisphosphate 3-kinase; CA: catalytic unit alpha; TKI: Tyrosine Kinase inhibitors; RAS: Rat sarcoma gene; RAF: rapidly accelerated fibrosarcoma; MEK: Mitogen-activated protein kinases; ERK: extracellular signalregulated kinases; SRC: sarcoma gene; FGFR1: Fibroblast growth factor receptor 1; Rb: Retinoblastoma; MAB: Monoclonal Antibody; PD: Pharmacodynamics; PK: Pharmacokinetics; Ig: Immunoglobulin; ADCC: antibody-dependent cellular cytotoxicity; HGF: hepatocyte growth factor; NeuGc-GM3: N-glycolylneuraminyl)-lactosylceramide antibody; IGF-1R: insulin-like growth factor-1 receptor; NK: natural killer; ADC: antibody-drug conjugate; Vc: volume of distribution; TF: Tissue Factor; CEA: carcinoembryonic antigen; CEACAM5: carcinoembryonic antigen-related cell adhesion molecule 5; RTK: receptor tyrosine kinase; AUC: area under the curve; ATP: Adenosine triphosphate; IND: Investigational New Drug; CDK: cyclin-dependent kinase; DNA: Deoxyribonucleic acid; JAK: Janus-associated kinases; STAT: signal transducer and activator of transcription; KIT: mast/ stem cell growth factor; FLT-3: FMS-like tyrosine kinase 3; TIE2: TEK tyrosine kinase; endothelial; TRKB: tropomyosin-related kinase B; AXL: Tyrosine-protein kinase receptor UFO; MAPK or MEK: mitogen-activated protein kinase; PDGFRb: platelet-derived growth factor receptor b; Lck: lymphocyte-specific protein tyrosine kinase; Lyn: Lck/Yes novel tyrosine kinase; CDC2: cyclin-dependent kinase 1; BTK: Bruton's tyrosine kinase; TEC: Tec protein tyrosine
\end{abstract}

kinase; $\mathrm{PIP}_{3}$ : phosphatidylinositol-3,4,5-trisphosphate; $\mathrm{BCR}$ : B-cell antigen receptor; TORC: TOR complex; mTOR: mammalian target of rapamycin; ATR: ataxia telangiectasia and rad3-related; Chk1: checkpoint kinase 1; CSF1R: colony-stimulating factor-1 receptor; wt: wild-type; EMT: epithelial-mesenchymal transition; TYRO3: TYRO3 protein tyrosine kinase; MER: Proto-oncogene tyrosine-protein kinase of MERTK gene; IDO1: indoleamine 2;3-dioxygenase; DCs: Dendritic cells; IFN: interferon; Tregs: tumor-associated regulatory $\mathrm{T}$ cells; ROS1: C-ros oncogene 1; PD-1: programmed death-1; PD-L1: programmed death- ligand 1; Vss: steady-state volume of distribution; Ig: Immunoglobulin; APCs: antigen presenting cells; CTLs: cytotoxic T-lymphocytes; CTLA4: cytotoxic T-lymphocyteassociated antigen-4; Fc: fragment constant; CDC: complementdependent cytotoxicity; TAAs: tumor-associated antigens; LAG-3: lymphocyte activation gene-3; TILs: tumor-infiltrating lymphocytes; MHC: major histocompatibility complex; IgSF: immunoglobulin superfamily; HLA: Human Leukocyte Antigen.

\section{Introduction/epidemiology}

Hepatocellular carcinoma (HCC) is the most common primary cancer of the liver. Liver cancer is the fifth common cause of cancer in men and seventh in women worldwide. It is the second most common cause of cancer death worldwide; however, the incidence is quite

${ }^{\star}$ Correspondence to: Timothy Allen, Global Allied Pharmaceuticals, Center for Excellence in Research and Development, 160 Vista Oak Dr. Longwood, FL 32779, USA, E-mail: timallenmed69@gmail.com

Key words: hepatocellular carcinoma (hcc), prognosis, monoclonal antibodies (mab), vaccines, mtor inhibitors, proteasome inhibitors,oncolytic virus therapy,cytokines, stat 3 inhibitors, check point inhibitors, adoptive t-cell therapy,a3 receptor inhibitors, myc inhibitors and kinase inhibitors

Received: January 09, 2019; Accepted: January 25, 2019; Published: January 28 2019 
different among different populations. It is rare in developed countries and prevalent in developing ones [1]. Approximately 500,000 deaths per year were reported annually. HCC accounts for $90 \%$ of all liver cancers [2].

According to the American Cancer Society,in 2014, it was found that about 33,190 new cases (24,600 in men and 8,590 in women) were diagnosed, and in the same year about 23,000 people $(15,870$ men and 7,130 women) died because of primary liver cancer and intrahepatic bile duct cancer in the United States [3].

Hepatocellular carcinoma is diagnosed at an average age of 63 years. More than $95 \%$ of people are diagnosed at the age of 45 years or older. About $3 \%$ are in between 35 and 44 years of age and approcimately $2 \%$ are younger than 35 [3]. In theUS, HCC is only $2 \%$ of all the reported cancers and the European Union has a low incidence with 8.29/100000 as compared to Asian and sub-Saharan African with incidence 120/100 000 , indicating that developing countries are considered to be in the dangerous zone for HCC $[2,4]$.

\section{Etiology/predisposing factors}

Approximately $90 \%$ of HCCs are associated with a known underlying risk factor. Such as:

\section{Viral infections}

Viral infections such as hepatitis B virus and hepatitis $\mathrm{C}$ virus are responsible for HCC. Hepatitis B is responsible for $50 \%$ cases of all HCC whilethe percentage for hepatitis $\mathrm{C}$ is $25 \%$. Hepatitis $\mathrm{C}$ is generally transmitted through blood products or infected needle or syringes, and hepatitis B infection is transmitted from mother to child, through sexual contact, sharing needles, syringes or other equipments to inject drugs, piercing and tattoos [5].

\section{Toxic substances}

Most common toxic substance for HCC is aflatoxins which are ingested through contaminated rice or peanuts.

\section{Alcohol}

Alcohol intake increases the risk of developing HCC.

\section{Metabolic disorders}

All metabolic diseases that finally lead to liver cirrhosis are considered as potential risk factors for Hepatocellular Carcinoma. Some of the diseases are diabetes, non-alcoholic, fatty liver disease and hereditary haemochromatosis.

\section{Immune-related factors}

Immune-related factors such as primary biliary cirrhosis and autoimmune hepatitis also causes HCC. Autoimmune hepatitis is characterized by a progressive destruction of the liver parenchyma, responsible for fibrosis and liver cirrhosis.

\section{Iron overloaded}

When total body iron is more than $5 \mathrm{~g}$, then it leads to the denaturation of the storage protein and further resulting in releasing large amounts of iron into the cytoplasm of the hepatocytes [6].

\section{HIV Coinfection}

HIV coinfection shortens the survival of patients with $\mathrm{HCV}$ (Hepatitis $\mathrm{C}$ virus) related cirrhosis [7].

\section{Pathophysiology and molecular basis}

Hepatocellular carcinogenesis is a slow process during which genomic changes progressively alter the hepatocellular phenotype to produce cellular intermediates that evolve into hepatocellular carcinoma. Several signaling cascades have been consistently found deregulated in HCC (e.g., WNT- $\beta$-catenin, PI3K/AKT/MTOR, RAS/ MAPK, IGF, HGF/MET, VEGF, EGFR, and PDGF).

Signaling pathways related to hepatocarcinoma

\section{MAPK pathway (RAS/RAF/MEK/ERK)}

Raf kinase inhibitor protein is a suppressor of the Raf/MAPK/ERK pathway. When its expression is suppressed by hepatitis B X protein and the hepatitis $\mathrm{C}$ core protein, up-regulated activation of the Raf/MAPK/ ERK signalling pathway happens which may lead to HCC [8].

\section{PI3K/AKT/MTOR pathway}

When growth factors, such asIGF and EGF bind to their respective receptors, PI3K is resultantly activated. It leads to PI3K induced formation of lipid second messenger PIP3b (phospho-inositol triphosphate) and it activates the serine/threonine kinase AKT. Activated AKT phosphorylate mTOR and BCL-2-associated death promoter. The activation of mTOR increases cellular proliferation. Phosphatase and tensin homolog (PTEN) is a tumour suppressor gene on chromosome 10, which de-phosphorylase the lipid products of PI3K in normal tissue [8]. In HCC, abnormality in the PTEN function results in the PI3K/AKT/mTOR over activation. The expression of PTEN is reduced in almost half of the cases of HCC tumors. This leads to the activation of above mentioned pathways. Reduced expression of PTEN has been associated with increased tumor grade, advanced stage of disease and reduction in the overall survival [9].

\section{VEGF/VEGFR, PDGFR, and FGFR}

Various angiogenic factors such as VEGFs, PDGFs, TGF-alpha and -beta, basic FGF, EGF, hepatocyte growth factor (HGF), angiopoietins and interleukin-4 and -8 are responsible for HCC growth and metastasis by complex alterations. Angiogenic signaling is induced by these growth factors and cytokines by various mechanisms,including activation of the RAF/MEK/ERK, PI3K/AKT/mTOR and JAK/signal transducer and activator pathways [8].

\section{EGFR, IGF and HGF/c-MET signaling}

EGFR has intracellular tyrosine kinase domain to trigger signal transduction through the MAPK and PI3K/Akt/mTOR pathways. It has been reported that EGFR is over expressed in case of HCC. In animmunohistochemical analysis, conducted by Buckley et al., the expression of EGFR and gene copy number in HCC was examined and its relationship was evaluated with clinicopathologic features, It was observed that EGFR was over expressed in 50 (66\%) of 76 HCCs; and fluorescence in situ hybridization (FISH) showed EGFRgene copies in $17(45 \%)$ of 38 HCCs $[9,10]$. However, such overexpression is widely seen in HCC that arises from cirrhotic liver, but no correlation has been established with other clinicopathologicfroms of the disease. Hence EGFR expression can be associated with high stage, proliferation, differentiation, invasion through vascular or hepatic route, recurrence and metastasis. It has also been postulated that in HCC, expression of EGFR is not the only predictor of the survival [11-16].

IGF-1R is a ligand gated autophosphorylationreceptor, which leads to activation of PI3K, protein kinase $\mathrm{B}$ and the RAF/MEK/ERK 
pathway. It has been reported thatoverexpression of IGF-2 occurs in $16 \%-40 \%$ of human HCCs [17].

HGF/Met pathway is one of the causes of tumor growth, invasion and angiogenesis in cancer. In this pathway, HGF activatesc-MET (a tyrosine kinase receptor) and the c-MET activatesphospholipase C, $\mathrm{PI} 3 \mathrm{~K}$ and ERK. It has been demonstrated that c-MET overexpression occurs in $20 \%-48 \%$ of HCC, which is supported by decreased 5 -year survival in HCC patients [18].

\section{WNT-BETA-CATENIN pathway}

WNTsignaling is responsible for decreased phosphorylation of $\beta$-catenin and $\beta$-catenin degradation,which further gets deposited in the cytoplasm and is shifted into the nucleus. Then it causes abnormal cellular proliferation and expresses membrane proteins involved in HCC, metastatic behavior, and cancer stem cells. Various studies have exhibited that HCV patients show $\beta$-catenin mutations (nearly $40 \%$ ) and HBV patients show $\beta$-catenin activation, by the expression of $\mathrm{HBx}$ protein [19].

\section{JAK/STAT pathway}

Janus tyrosine kinases (Jak1, 2 and 3 and Tyk2), is activated by cytokines.Further,this Janus tyrosine kinases activates Stat1-6. Higher levels of activated Stat1, Stat3, and Stat5 have been reported in tumors compared with surroundingliver tissue [8].

\section{P53/ARF pathway}

The p53/ARF pathway has a function as cell cycle regulation, apoptosis and DNA repair. Cellular stress is responsible to initiate this pathway. The p53 network also involves in fundamental cellular signals, such as the Wnt/b catenin, RB/INK4a and p38 MAP pathways. As the p53/ARF pathway plays a vital role in the maintenance of various cellular responses, disturbances in this pathway may be responsible for the progression of various cancers, including HCC [20].

\section{RB/INK4A pathway}

Role of RB/INK4A is to regulate G1-S phase of cell cycle progression. The activities of $\mathrm{RB}$ have been associated with the phosphorylation of this molecule, which in turn, leads to the progression of cell cycle along with $\mathrm{CDK}$ activation. The RB, before getting phosphorylated, adheres to the transcription factor E2F-1, which interferes with its interaction with transcriptional machinery of the cell. After phosphorylation, RB sequesters E2Fs, which are responsible for various gene transcriptions. Out of these transcripted genes, cyclin $\mathrm{E}$ adheres to the CDK2 and induces replication of DNA during the cell cycle. In HCC, the members of this pathway are frequently aberrant. It has been reported that the components of this pathway are affected in approximately $81 \%$ of the cases of HCC [21].

\section{Myc}

It is an important oncogene, which is up-regulated in several human cancers and hence, portrays the phenomenon of "oncogene addiction". It is responsible for the induction of telomerase activity that aids the process of hepatocarcinogenesis and is also found to be involved in the development of HCC. In HCC, the inactivated Myc is responsible for the differentiation of small population of cells, while the other cells remain dormant s and produce phenotypically diverse populations of tumor and possibly the origin of CSCs [22].

\section{miRNAs}

miRNAs are a group of small non-coding RNAs, that are involved in post transcriptional gene regulation and the deregulated expression of miRNAs has been suggested to modulate several molecular signaling pathways that lead to hepatocellular carcinogenicity. miR-26 expression has been found to be associated with HCC gender disparity. Moreover, overexpression of miR-21 is responsible for the loss of hetrogygosity at the PTEN locus, which results in the activation of Akt pathway and aids tumorigenesis. In the same manner, HCC-associated miRNAs have also been identified, which are responsible for regulation of cell cycle and inhibition of apoptosis [22].

\section{Immunotherapy}

A. Kinase inhibitors

a. FDA approved kinase inhibitors

\section{Sorafenib}

\section{Indications and use}

Sorafenib is a kinase inhibitor and has been received FDA approval for the treatment of unresectable hepatocellular carcinoma [23].

\section{Contraindications}

It is contraindicated in patients with known severe hypersensitivity to Sorafenib.

\section{Warnings}

Cardiac Ischemia and/or Infarction, bleeding, hypertension, dermatologic toxicities, gastrointestinal perforation, wound healing complication, risk of QT interval prolongation, drug induced hepatitis, embryo fetal risk.

\section{Adverse Events}

Most commonly are diarrhea, fatigue, infection, alopecia, handfoot skin reaction, rash, weight loss, decreased appetite, nausea, gastrointestinal and abdominal pain, hypertension, and hemorrhage.

Non-FDA Approved Kinase Inhibitors: Few other kinase inhibitorsthat are under clinical trials phase I, II, and III as mentioned in the Table 1

\section{mTOR Inhibitors}

\section{FDA Approved mTORInhibitors}

\section{Sirolimus}

A natural macrocyclic lactone produced by the bacterium Streptomyces hygroscopicus, with immunosuppressant properties [27]. In cells, sirolimus binds to the immunophilin FK Binding Protein-12 (FKBP-12) to generate an immunosuppressive complex

Table 1. Non-FDA approved TKIs [24-26]
\begin{tabular}{|c|c|c|c|c|}
\hline $\begin{array}{c}\text { Kinase } \\
\text { inhibitors }\end{array}$ & $\begin{array}{c}\text { Clinical trial } \\
\text { identifier no. }\end{array}$ & PHASE & Study Design & Target \\
\hline $\begin{array}{c}\text { Tivantinib (ARQ } \\
197)\end{array}$ & NCT00802555 & Phase I & $\begin{array}{c}\text { Safety Study, } \\
\text { Open Label }\end{array}$ & c-Met \\
\hline Foretinib & NCT00920192 & Phase I & $\begin{array}{c}\text { Safety/Efficacy } \\
\text { Study, Open } \\
\text { Label }\end{array}$ & MET/VEGFR \\
\hline Tivozanib & NCT01835223 & Phase I/II & $\begin{array}{c}\text { Efficacy Study, } \\
\text { Open Label }\end{array}$ & VEGFR \\
\hline
\end{tabular}


that binds to and inhibits the activation of the mammalian Target Of Rapamycin (mTOR), a key regulatory kinase. This results in inhibition of $\mathrm{T}$ lymphocyte activation and proliferation that occurs in response to antigenic and cytokine (IL-2, IL-4, and IL-15) stimulation and inhibition of antibody production.

\section{Indications and use}

It is generally used as prophylaxis during rejection in patients with renal transplants. But new studies prove its use for liver transplant in HCC as it can improve recurrence, survival rates after it.

\section{Contraindications}

It is contraindicated in patients with hypersensitivity to sirolimus.

\section{Warnings}

Dyslipidemia, combination with CSA and steroids, hepatic artery thrombosis.

\section{Adverse Events}

Main side effects are infections and lymphoma, impaired wound healing, hypercholesterolemia and hypertriglyceridemia ( $50 \%$ of pts), hypertension, thrombocytopenia, arthralgia, acne and rash, insomnia and tremor.

\section{Non-FDA Approved mTORInhibitors}

The mTOR inhibitors that are under clinical trials are given below

\section{Everolimus}

A derivative of the natural macrocyclic lactone sirolimus with immunosuppressant and anti-angiogenic properties. In cells, everolimus binds to the immunophilin FK Binding Protein-12 (FKBP12) to generate an immunosuppressive complex that binds to and inhibits the activation of the mammalian Target of Rapamycin (mTOR), a key regulatory kinase. Inhibition of mTOR activation results in the inhibition of $\mathrm{T}$ lymphocyte activation and proliferation associated with antigen and cytokine (IL-2, IL-4, and IL-15) stimulation and the inhibition of antibody production.

\section{Temsirolimus}

An ester analog of rapamycin. Temsirolimus binds to and inhibits the mammalian target of rapamycin (mTOR), resulting in decreased expression of mRNAs necessary for cell cycle progression and arresting cells in the G1 phase of the cell cycle [29]. mTOR is a serine/threonine kinase which plays a role in the PI3K/AKT pathway that is upregulated in some tumors. This Phase II trial is being developed following the completion of a Phase I study of the combination of temsirolimus and sorafenib in 25 first-line therapy patients with advanced hepatocellular carcinoma.

\section{Monoclonal Antibodies}

\section{Non-FDA Approved Monoclonal Antibodies.}

The mAbs that are under clinical trials are given in Table 2.

\section{Vaccine-Based Therapy:}

\section{Non-FDA Approved Vaccines [39-44]} below:

The vaccine based therapies that are under clinical trials are given

\section{NY-ESO-1 reactive TCR}

Human autologous peripheral blood lymphocytes (PBLs) transduced with a retroviral vector, encoding a T cell receptor (TCR) specific for the cancer-testis antigen NY-ESO-1, with potential antineoplastic activity.

\section{DCVax-Direct}

This cancer vaccine consisting of lymphocytes, which are harvested from a patient with lung cancer.

\section{Fang}

Autologous tumor cells transfected with a plasmid expressing recombinant human granulocyte macrophage-colony stimulating factor (rhGM-CSF) and bifunctional short hairpin RNA (bi-shRNA) against furin, with potential immunostimulatory and antineoplastic activities.

\section{DEC-205/NY-ESO-1 fusion protein CDX-1401}

A fusion protein consisting of a fully human monoclonal antibody directed against the endocytic dendritic cell (DC) receptor, DEC-205, linked to the tumor-associated antigen (TAA) NY-ESO-1 with potential immunostimulating and antineoplastic activities.

\section{Tumor cell-derived DRibbles vaccine}

An autophagosome-enriched cancer vaccine composed of shortlived proteins (SLiPs) and defective ribosomal products (DRiPs) derived from tumor cells, with potential immunostimulating and antineoplastic activities.

\section{COMBIG-DC}

A cancer vaccine consisting of allogeneic, immortalized dendritic cells (DCs) loaded with tumor specific antigens and activated, with

Table 2. Non-FDA approved monoclonal antibodies [30-38]

\begin{tabular}{|c|c|c|c|c|}
\hline mAbs & Clinical trial identifier no. & Phase & Study Design & Target \\
\hline Bevacizumab & NCT00365391 & Phase II & $\begin{array}{c}\text { Efficacy Study, } \\
\text { Single Group Assignment, } \\
\text { Open Label }\end{array}$ & VEGF \\
\hline IMMU-132 & NCT01631552 & Phase $1 / 2$ & Safety/Efficacy Study, open label & Trop-2 \\
\hline TRC 105 & NCT01306058 & Phase I/II & Safety/Efficacy Study, open label & endoglin (CD105) \\
\hline MORAb-004 & NCT01748721 & Phase I & Safety Study, open label & endosialin/TEM1 \\
\hline MEDI4736 & NCT01693562 & Phase I/II & $\begin{array}{l}\text { Non-Randomized, Safety/ } \\
\text { Efficacy Study, open label }\end{array}$ & B7H1 \\
\hline Mapatumumab & NCT00712855 & Phase I & Safety Study, Open Label & TRAIL-R1 \\
\hline Cixutumumab & NCT01008566 & Phase I & Safety Study, Open Label & IGF-1R \\
\hline Bavituximab & NCT01264705 & Phase I/II & Safety/Efficacy Study, OpenLabel & $\mathrm{ADCC}$ \\
\hline Ipilimumab & NCT01658878 & Phase I/II & Safety/Efficacy Study, OpenLabel & $\mathrm{ADCC}$ \\
\hline
\end{tabular}


potential immunostimulatory and antineoplastic activities. Upon intratumoral administration of the allogeneic dendritic cell vaccine COMBIG-DC, these activated DCs attract natural killer (NK) cells, induce an anti-inflammatory response leading to the induction of NK-cell-mediated tumor cell death. Upon release of tumor associated antigens (TAAs) from the lysed tumor cells, these antigens are taken up by antigen presenting cells which activate the immune system to elicit a potent cytotoxic T-cell (CTL) response against the TAAs, resulting in the death of TAAs-expressing tumor cells.

\section{Adoptive cell therapy}

\section{Non-FDA Approved Adoptive T Cell Therapy}

There are some adoptive $\mathrm{T}$ cell therapies that are not currently approved by FDA for HCC [45-48]. However, these treatment modalities are under clinical trials in phase I, II, and III as given below:

\section{TIL}

Autologous peripheral blood lymphocytes-derived $\mathrm{T}$ cells transduced with a retroviral encoding TIL 1383I, a T cell receptor (TCR) specific for melanoma antigen tyrosinase, with potential immunostimulating and antineoplastic activity.

\section{Anti-NY ESO-1 mTCR PBL} [45].

It represent potential targets for T-cell receptor (TCR) gene therapy

\section{Anti-MAGE-A3-DP4 TCR PBL}

Human autologous T-lymphocytes transduced with a retroviral vector encoding a T-cell receptor (TCR) specific for the human leukocyte antigen (HLA)- DP0401/0402-restricted, melanoma antigen A3 (MAGE-A3), with potential antineoplastic activity.

\section{Anti-VEGFR2 CAR CD8 plus PBL}

Phase I/II Study of Metastatic Cancer Using lymphodepletingco nditioningFollowed by Infusion of Anti-VEGFR2 Gene Engineered CD8+ Lymphocytes is ongoing.

\section{Cytokines}

\section{Non-FDA Approved Cytokines}

There are some cytokines that are not currently approved by FDA for HCC $[49,50]$.

However, these cytokines are under clinical trials in phase I, II, and III as given below:

\section{rh IL-15}

Recombinant Human IL-15 is an immunomodulating cytokine that stimulates the proliferation of $\mathrm{T}$ lymphocytes.

\section{NHS-IL 12}

A fusion protein consisting of the heavy-chains of the human antibody NHS76, raised against DNA released by necrotic tumor cells, and fused to two molecules of a genetically modified human interleukin-12 (IL-12) with potential immunostimulating and antineoplastic activities.

\section{Oncolytic Virus Therapy}

This therapy act by using a modified virus that can cause tumor cells to self-destruct and generate a greater immune response against the cancer [51].

\section{HSV-1 rRp 450}

A gene therapy agent containing an attenuated, replicationcompetent, genetically engineered mutant form of the Herpes simplex virus 1 (HSV-1) strain KOS with potential antineoplastic activity.

\section{Proteasome Inhibitors}

\section{Oprozomib}

A proteasome inhibitor with potential antineoplastic activity [52]. Oprozomib inhibits the activity of the proteasome, thereby blocking the targeted proteolysis normally performed by the proteasome; this may result in an accumulation of unwanted or misfolded proteins. Disruption of various cell signaling pathways may follow, eventually leading to the induction of apoptosis and inhibition of tumor growth.

\section{STAT3 Inhibitors}

\section{OPB-31121}

An inhibitor of signal transducer and activator of transcription 3 (STAT3), with potential antineoplastic activity. OPB-31121 inhibits the phosphorylation of STAT3, which prevents binding of STAT3 to DNA sequences on a variety of STAT3-responsive promoters and may result in the inhibition of STAT3-mediated transcription and, potentially, the inhibition of tumor cell proliferation [53]. STAT3 is constitutively activated in a variety of cancers, contributing to the loss of cell growth control and neoplastic transformation.

\section{Checkpoint inhibitors}

Non-FDA Approved Checkpoint Inhibitors

The checkpoint inhibitors that are under clinical trials are given in Table 3.

\section{MycInhibitors}

\section{Non-FDA Approved MycInhibitors}

There are no Myc inhibitors that are currently approved by FDA for HCC [56].

However, some Myc inhibitors are under clinical trials.

\section{DCR-MYC}

A lipid nanoparticle-based formulation consisting of smallinterfering RNAs (siRNAs) directed against the oncogene c-Myc encapsulated in lipids with potential antineoplastic activity. Upon intravenous administration of MYC-targeting siRNA DCR-MYC, the lipid formulation promotes the uptake by tumor cells where the siRNAs moieties are subsequently released. The siRNAs bind to c-Myc mRNAs, which may result in the inhibition of translation and expression of the c-Myc protein and leads to growth inhibition for tumor cells that are overexpressing c-Myc. c-Myc, a proto-oncogene overexpressed in a variety of cancers, is involved in cellular proliferation, differentiation, and apoptosis. 
Table 3. Non-FDA approved checkpoint inhibitors [54,55]

\begin{tabular}{|c|c|c|c|c|}
\hline $\begin{array}{c}\text { Checkpoint } \\
\text { inhibitors }\end{array}$ & $\begin{array}{c}\text { Clinical trial } \\
\text { identifier no. }\end{array}$ & PHASE & Study Design & Target \\
\hline Pembrolizumab & NCT02054806 & Phase I & $\begin{array}{c}\text { Efficacy Study, } \\
\text { open label }\end{array}$ & PD-1 \\
\hline Tremelimumab & NCT01853618 & Phase I & $\begin{array}{c}\text { Non- } \\
\text { Randomized, } \\
\text { Safety Study, } \\
\text { open label }\end{array}$ & CTLA-4 \\
\hline
\end{tabular}

Table 4. Non-FDA approved A3 agonist receptor [57]

\begin{tabular}{|c|c|c|c|c|}
\hline Drug & $\begin{array}{c}\text { Clinical trial } \\
\text { identifier no. }\end{array}$ & Phase & Study Design & Target \\
\hline CF102 & NCT00790218 & Phase I/II & $\begin{array}{c}\text { Safety/Efficacy } \\
\text { Study, Open } \\
\text { Label }\end{array}$ & A3AR \\
\hline
\end{tabular}

\section{A3 Receptor Agonist}

Non-FDA Approved A3 Receptor Agonist (Table 4)

\section{Conclusion}

Hepatocellular carcinoma is the major type of liver cancer. Mostly affected zone of this type of cancer are developing countries. Its main causes are viral infections, such as HBV and HCV. Various cell signaling pathways are demonstrated to play a role in its molecular pathophysiology.

Immunotherapy has proven to be effective in the treatment of hepatocellular carcinoma. Immunotherapeutics include monoclonal antibodies, vaccines, adoptive cell therapy and cytokine. There is only one kinase inhibitor,sorafenib, and one mTOR inhibitor, sirolimus, that are FDA approved for the treatment of HCC,however, many immunotherapeutic agents are under clinical trials. Our success in treating hepatocellular carcinoma is increasing and advancing with the knowledge of the function of the immune system. Researchers are still challenged in exploring innate and adaptive immune systems. Immunotherapy has been a promising development in the past few years. The recent activities have increased our understanding of the tumor microenvironment, various immunotherapeutic modalities or combination therapy (like chemotherapy with immunotherapy). Additionally, the effects of such modalities in combination of immunotherapy in cancer patients are still in its exploratory phase. Proper preclinical and clinical designs are the important pillars in understanding the future of immunotherapy in treating cancer patients.

\section{References}

1. World Health Organization, 2014.

2. Jelic S, Sotiropoulos GC (2010) Clinical practice guidelines, hepatocellular carcinoma: ESMO Clinical Practice Guidelines for diagnosis, treatment and follow-up. Annals of Oncology 21: 59-64.

3. What are the key statistics about liver cancer? 2014.

4. Stuart KE, Anand AJ, Jenkins RL (1996) Hepatocellular carcinoma in the United States prognostic features, treatment outcome, and survival. Cancer 77: 2217-2222.

5. Sanyal AJ, Yoon SK, Lencioni R (2010) The etiology of hepatocellular carcinoma and consequences for treatment. Oncologist 4: 14-22. [Crossref]

6. Kew MC (2014) Hepatocellular carcinoma: epidemiology and risk factors. J Hepatocell Carcinoma 1: 115-125. [Crossref]

7. Hepatitis, 2014.

8. Shin JW, Chung YH (2013) Molecular targeted therapy for hepatocellular carcinoma: Current and future. World J Gastroenterol 19: 6144-6155.
9. Hu TH, Huang CC, Lin PR, Chang HW, Ger LP, et al. (2003) Expression and prognostic role of tumor suppressor gene PTEN/MMAC1/TEP1 in hepatocellular carcinoma. Cancer 97: 1929-1940. [Crossref]

10. Buckley AF, Burgart LJ, Sahai V (2008) Epidermal growth factor receptor expression and gene copy number in conventional hepatocellular carcinoma. Am J ClinPathol 129: 245-251.

11. Tang Z, Qin L, Wang X (1998) Alterations of oncogenes, tumor suppressor genes and growth factors in hepatocellular carcinoma: with relation to tumor size and invasiveness. Chin Med $J$ 111:313-318.

12. Ito Y, Takeda T, Sakon M (2001) Expression and clinical significance of Erb-B receptor family in hepatocellular carcinoma. Br J Cancer 84:1377-1383.

13. Zhao YN, Cao J, Wu FX, Ou C, Yuan WP (2004) Expression and significance of EGF mRNA and EGFR mRNA in hepatocellular carcinoma. Ai Zheng 23: 762-766. [Crossref]

14. Harada K, Shiota G, Kawasaki H (1999) Transforming growth factor alpha and epidermal growth factor receptor in chronic liver disease and hepatocellular carcinoma. Liver 19: 318-325.

15. Kira S, Nakanishi T, Suemori S (1997) Expression of transforming growth factor alpha and epidermal growth factor receptor in human hepatocellular carcinoma. Liver 17: 177-182.

16. Hamazaki K, Yunoki Y, Tagashira H (1997) Epidermal growth factor receptor in human hepatocellular carcinoma. Cancer Detect Prev 21: 355-360.

17. De Souza AT, Hankins GR, Washington MK (1995) Frequent loss of heterozygosity on $6 \mathrm{q}$ at the mannose 6-phosphate/insulin-like growth factor II receptor locus in human hepatocellular tumors. Oncogene 10: 1725-1729.

18. Tavian D, De Petro G, Benetti A (2000) u-PA and c-MET mRNA expression is coordinately enhanced while hepatocyte growth factor mRNA is downregulated in human hepatocellular carcinoma. Int J Cancer 87: 644-649.

19. Zhang Y, Wei W, Cheng N (2012) Hepatitis C virus-induced up-regulation of microRNA-155 promotes hepatocarcinogenesis by activating Wnt signaling. Hepatology 56: 1631-1640.

20. Nishida N, and Goel A(2011) Genetic and epigenetic signatures in human hepatocellular carcinoma: a systematic review. Current Genomics 12: 130-137.

21. Azechi H, Nishida N, Fukuda Y (2001) Disruption of the p16/cyclin D1/retinoblastoma protein pathway in the majority of human hepatocellular carcinomas. Oncology 60 346-354.

22. Kumar M, Zhao X, Wang XW (2011) Molecular carcinogenesis of hepatocellular carcinoma and intrahepatic cholangiocarcinoma: one step closer to personalized medicine. Cell \& Bioscience 1: 1-13.

23. FDA Label Sorafenib (NEXAVAR). Marketed by: Onyx Pharmaceuticals.

24. ArQule; ArQule. Safety study of arq 197 in cirrhotic patients with hepatocellular carcinoma (HCC). In: ClinicalTrials.gov. Bethesda (MD): National Library of Medicine (US).

25. GlaxoSmithKline; GlaxoSmithKline. Safety study of foretinib (gsk1363089) in adults with liver cancer. in: ClinicalTrials.gov. Bethesda (MD): National Library of Medicine (US)

26. Roswell park cancer institute; national cancer institute (nci). tivozanib in treating patients with liver cancer that is metastatic or cannot be removed by surgery. in ClinicalTrials.gov. Bethesda (MD): National Library of Medicine (US).

27. FDA label rapamune (sirolimus). marketed by manufactured for: wyeth pharmaceuticals inc. philadelphia

28. National Health Research Institutes, Taiwan; Novartis. randomized phase i/ii of rad001 in advanced hepatocellular carcinoma (HCC). In: ClinicalTrials.gov. Bethesda (MD): National Library of Medicine (US).

29. National Cancer Institute (NCI); Bevacizumab and erlotinib in treating patients with advanced liver cancer.in: ClinicalTrials.gov. Bethesda (MD): National Library of Medicine (US).

30. https://clinicaltrials.gov/ct2/show/NCT01687673

31. Immunomedics, Inc.; immunomedics, inc. phase i/ii study of immu-132 in patients with epithelial cancers. in: ClinicalTrials.gov. Bethesda (MD): National Library of Medicine (US).

32. National Cancer Institute (NCI); National Cancer Institute (NCI). Sorafenib and TRC105 in hepatocellular cancer. In: ClinicalTrials.gov. Bethesda (MD): National Library of Medicine (US). 
33. Morphotek; morphotek. morab-004 in treating young patients with recurrent or refractory solid tumors or lymphoma. in: ClinicalTrials.gov. Bethesda (MD): National Library of Medicine (US).

34. MedImmune LLC; MedImmune LLC.A phase 1/2 study to evaluate medi4736.in: ClinicalTrials.gov. Bethesda (MD): National Library of Medicine (US).

35. Human Genome Sciences Inc., a GSK Company; GlaxoSmithKline. A study of mapatumumab in combination withsorafenib in subjects with advanced hepatocellular carcinoma (HCC). In: ClinicalTrials.gov. Bethesda (MD): National Library of Medicine (US).

36. National Cancer Institute (NCI); National Cancer Institute (NCI). Cixutumumab and sorafenibtosylate in treating patients with advanced liver cancer. in: ClinicalTrials.gov. Bethesda (MD): National Library of Medicine (US).

37. University of Texas southwestern medical center; university of texas southwestern medical center. study of bavituximab and sorafenibin patients with advanced liver cancer. In: ClinicalTrials.gov. Bethesda (MD): National Library of Medicine (US).

38. https://clinicaltrials.gov/ct2/show/NCT01658878

39. Jonsson comprehensive cancer center; jonsson comprehensive cancer center.gene and vaccine therapy in treating patients with advanced malignancies. In: ClinicalTrials.gov. Bethesda (MD): National Library of Medicine (US).

40. Northwest biotherapeutics; northwest biotherapeutics. safety and efficacy study of dcvax-direct in solid tumors.in: ClinicalTrials.gov. Bethesda (MD): National Library of Medicine (US).

41. Gradalis, Inc.; Gradalis, Inc. trial of bi-shrna-furin and granulocyte macrophage colony stimulating factor (gmcsf) augmented autologous tumor cell vaccine for advanced cancer (fang). in: ClinicalTrials.gov. Bethesda (MD): National Library of Medicine (US).

42. Roswell park cancer institute; roswell park cancer institute. vaccine therapy with or without sirolimus in treating patients with ny-eso-1 expressing solid tumors. in: ClinicalTrials.gov. Bethesda (MD): National Library of Medicine (US).

43. National Cancer Institute (NCI); Tumor cell vaccines and iscomatrix with chemotherapy after tumor removal. in: ClinicalTrials.gov. Bethesda (MD): National Library of Medicine (US).

44. Immunicum AB; Uppsala university. phase i safety study of dendritic cell vaccine to treat patients with hepatocellular carcinoma. in: ClinicalTrials.gov. Bethesda (MD): National Library of Medicine (US)

45. National Cancer Institute (NCI); Immunotherapy using tumor infiltrating lymphocytes for patients with metastatic cancer. in: ClinicalTrials.gov. Bethesda (MD): National Library of Medicine (US).
46. National Cancer Institute ( $\mathrm{NCI}$ ); National cancer institute (nci). t cell receptor immunotherapy targeting ny-eso-1 for patients with ny-eso- 1 expressing cancer. in: ClinicalTrials.gov. Bethesda (MD): National Library of Medicine (US).

47. National Cancer Institute (NCI); T cell receptor immunotherapy targeting mage-a3 for patients with metastatic cancer who are hla-dp0401 positive. in: ClinicalTrials.gov. Bethesda (MD): National Library of Medicine (US).

48. National Cancer Institute (NCI); National cancer institute (nci). car T cell receptor immunotherapy targeting vegfr2 for patients with metastatic cancer. in: ClinicalTrials. gov. Bethesda (MD): National Library of Medicine (US).

49. National Cancer Institute (NCI); Continuous infusion of rhil-15 for adults with advanced cancer. in: ClinicalTrials.gov. Bethesda (MD): National Library of Medicine (US).

50. National Cancer Institute (NCI); NHS-IL12 for solid tumors.in: ClinicalTrials.gov. Bethesda (MD): National Library of Medicine (US).

51. Massachusetts General Hospital. rRp450-Phase I trial in liver metastases and primary liver tumors. in: ClinicalTrials.gov. Bethesda (MD): National Library of Medicine (US).

52. Onyx Therapeutics, Inc.; onyx therapeutics, inc. phase $1 \mathrm{~b} / 2$ study of oprozomib in combination withsorafenib in subjects with advanced hepatocellular carcinoma. in: ClinicalTrials.gov. Bethesda (MD): National Library of Medicine (US).

53. Otsuka Pharmaceutical Co., Ltd.; otsuka pharmaceutical co., ltd. phase i/ii study of opb-31121 in patients with progressive hepatocellular carcinoma. in: ClinicalTrials. gov. Bethesda (MD): National Library of Medicine (US).

54. Merck Sharp \& Dohme Corp; Merck sharp \& dohme corp. study of pembrolizumab (mk-3475) in participants with advanced solid tumors (mk-3475-028/keynote-28). in: ClinicalTrials.gov. Bethesda (MD): National Library of Medicine (US).

55. National Cancer Institute (NCI); Tremelimumab with chemoembolization or ablation for liver cancer. in: ClinicalTrials.gov. Bethesda (MD): National Library of Medicine (US).

56. Dicerna Pharmaceuticals, Inc., Dicerna pharmaceuticals, inc. phase ib/2, multicenter, dose escalation study of dcr-myc in patients with hepatocellular carcinoma. in: ClinicalTrials.gov. Bethesda (MD): National Library of Medicine (US).

57. Can-FiteBioPharma; A phase 1-2 study of cf102 in patients with advanced hepatocellular carcinoma. in: ClinicalTrials.gov. Bethesda (MD): National Library of Medicine (US)

Copyright: (C2019 Allen T. This is an open-access article distributed under the terms of the Creative Commons Attribution License, which permits unrestricted use, distribution, and reproduction in any medium, provided the original author and source are credited. 